\title{
EINE MÖGLICHE NEUINTERPRETATION DES ÄGYPTISCHEN DRAMATISCHEN RAMESSEUMPAPYRUS 45, SZENE 12
}

\author{
STEFAN BOJOWALD
}

\author{
Ägyptologisches Seminar der Universität Bonn \\ Regina-Pacis-Weg 7. 53113 Bonn, Deutschland \\ e-mail: stefan.bojowald@t-online.de
}

In this paper, a new approach of interpreting Egyptian Dramatic Ramesseumpapyrus 45, Scene 12, is undertaken. In the concrete case a new explanation for $s$ in of K. Sethe, who translates the whole passage as 'to kill goose' or $s$ alone with 'to strangulate goose', is questioned. The new proposal tries to prove that $s$ can be a defective writing for śt $t$ 'pluck' or $\underline{t} s \dot{i}$ 'bind', respectively. The exchange between $s$ and dentals may have played a vital role.

Key words: Egyptian philology, Dramatic Ramesseum Papyrus 45:12, new explanation for $\dot{s}$ in $\ln \rightarrow \rightarrow-$

In Dramatischer Ramesseumpapyrus 45, Szene 12 ist im Rahmen der Vorbereitungen für das hnk.t 'Opfer' die Halbzeile (Sethe 1964, S. 147) zu finden, die Sethe mit 'Gänse ${ }^{1}$ schlachten' wiedergeben will. Der vorliegende Artikel setzt sich die Erklärung von $s ́$ zum Ziel, für das von Sethe bereits zwei Alternativen vorgeschlagen worden sind. Im ersten Fall hat er die Möglichkeit eines Kausativpräfixes in Erwägung gezogen (Sethe 1964, S. 152). Die Wahl begründet er damit, dass insbesondere im Arabischen von Nomina Kausative gebildet werden können, die dadurch zum Objekt oder Zielpunkt der betreffenden Handlung werden. Der gleiche Kausativgebrauch wird von ihm auch für das Ägyptische unterstellt, was in Verbindung mit der śmn 'Gans' ein denominatives Verb śsmn 'Gänse töten' ergeben würde. Die Bedeutung hat zwar so auch ihren Weg ins Wörterbuch (WB IV, S. 277, Lemma 8) gefunden, wo sie aber vorsorglich durch ein Fragezeichen markiert worden ist. Im zweiten Fall hat Sethe ein eigenes Verb ś mit der Bedeutung 'erdrosseln o. ä.' angenommen (Sethe 1964, S. 153).

${ }^{1}$ Zur Verwendung der śmn 'Gans' im hnkk.t 'Opfer' vgl. auch Sethe (1934, S. 384, Spruch 326-435: §§ 534-787). Die Schreibung der śmn 'Gans' mit stimmlosem $s$ kommt auch bei de Buck (1957, Vol. III, Paragraph 130g) und Jansen-Winkeln (2007, S. 140, vor). 
Die Erklärung stößt jedoch auf die Schwierigkeit, dass die Bedeutung 'erdrosseln' für $s$ an keiner anderen Stelle belegt ist. Im Anschluss wird sich zeigen, dass sie sehr wahrscheinlich aufgegeben werden muss. In den folgenden Zeilen soll daher eine erneute Annäherung an dieses Wort gewagt werden.

In solchen Situationen kann immer zuerst geprüft werden, ob die übrigen Bedeutungen des Wortes die Suche nach der verlangten Bedeutung unterstützen können. Die Methode stellt sich aber im vorliegenden Fall als untauglich heraus, da die Schreibungen $s$ (Gardiner 1935, Pl. 38, vso 7, 4) für $s ̌ w$ 'Schu', ś (WB IV, S. 191, Belegschreibungen) für śr 'Gans', ś (Koenig 1979, S. 118; Quack 1999, S. 161) für sí 'Mann' und ś (Černý 1939, S. 62a; Vernus 1980, S. 130e; Jansen-Winkeln, 2001, S. 74 27) für śnb 'genesen/gesund sein' für das Verständnis nicht gerade förderlich sind.

Im Folgenden werden jedoch zwei Schreibungen zur Kenntnis gegeben, mit denen der Sache schon sehr viel näher gekommen wird. Das erste Beispiel ist die Schreibung ś (El-Kholi, 2006, S. 68-69) für śd im Fischnamen śd-ḥw, bei welcher der zweite Radikal $d$ am Ende des Wortes weggefallen ist. Die Elision des $d$ dürfte sich erst nach der Assimilation an $s$ ereignet haben. Die Voraussetzung hierfür war sicher der Austausch von $s$ mit spirantisierten oder entspirantisierten Dentalen, dessen Existenz schon wiederholt festgehalten worden ist (Sethe 1899, S. 164; Fecht 1981, S. 149; KRI V, 201, 6; Westendorf 1962, S. 41). Das zweite Beispiel stellt die Schreibung $s$ (Janssen, 2008, S. 22) für $s \underline{d} r$ in der Textilbezeichnung $i f d \underline{s} \underline{d} r^{2}$ dar. Die Schreibung ist hinsichtlich der Elision des Dentals bei $s$ mit demselben Phänomen wie die Schreibung $s$ für $s \underline{d}$ zu begründen. Die hier ebenfalls zu beobachtende Elision von $r$ ist schon mehrere Male vorgekommen (vgl. einstweilen Fecht 1960, S. 34, 140, 149 ff). Die spezielle Elision von $r$ nach $s$ hatte sich bereits bei der oben behandelten Schrei-

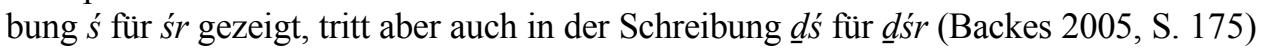
'Wege bahnen' auf. Da die Worte $\underline{d} s^{\prime} r$ und $\underline{d} s$ aus denselben drei Konsonanten bestehen, wären die Elisionen bei ihnen nur unterschiedlich weit fortgeschritten. Das Wortspiel zwischen $\dot{s} \underline{d}$ zerbrechen und $\dot{s} \underline{d} r$ 'schlafen' (Otto 1960, S. 54, Point 6) könnte hier ebenfalls ergänzend genannt werden.

Der Austausch von $s$ mit Dentalen soll auch bei der Erklärung von $s ́$ im obigen Beispiel eine zentrale Rolle spielen. Wenn diese Vorgehensweise gewählt wird, kommen zwei Verben in den Sinn, mit denen die Lösung des Problems in greifbare Nähe rücken könnte.

Das erste Verb lautet śtt (WB IV, S. 363, Lemma 1) 'rupfen', das in einer Handvoll Beispiele bezeugt ist. Das erste Beispiel steht in der 'Lehre vom reiselustigen Sohn' (oOIC ${ }^{3}$ 12074, vso 7-8), der sich nach seiner unrühmlichen Rückkehr aus dem Ausland vom Vater die vorwurfsvollen Worte $t w=k$ s $\underline{t} t \underline{i} \underline{t}$. $. w i h . t=k / t w=k$ wij $w i$ ? $m-b 3 h=i$ (Cerny-Gardiner 1957, P1. LXXIX) 'Du bist gerupft ${ }^{4}$, fortgenommen sind

${ }^{2} \mathrm{Zu}$ dieser Textilbezeichnung vgl. auch Helck (1965, S. 926-928).

${ }^{3}$ oOIC $=$ Ostrakon Oriental Institute Chicago.

${ }^{4}$ Die von Brunner (1958, S. 196), für śst vorgeschlagene Bedeutung 'betrügen' ist von Guglielmi (1983, S. 159) nicht unwidersprochen hingenommen worden, die eine übertragene Bedeutung 'ausplündern' vermutet hat. 
deine Sachen. Jämmerlich ${ }^{5}$ stehst du nun vor mir. ${ }^{6}$ anhören muss. Das zweite Beispiel dieses Wortes ist im Vergleich eines auf einem Feldzug nach Syrien in Bedrängnis geratenen Soldaten mit einem nackt und kahl gerupften Vogel $\left(\right.$ pAnast $^{7}$ III 5,12 ) zu finden, indem das strapaziöse Leben dieses Infanteristen mit den Worten ś $p r=f p 3$ hril, iw $=f$ mi 3pd śttt, iw nn wn ph.ti $m$ h3.wt $=f$ (Gardiner 1937, S. 26) 'Hat er das Schlachtfeld erreicht, ist er wie ein gerupfter Vogel, da in seinem Körper keinerlei Kraft mehr ist'. (Caminos 1954, S. 92) beschrieben wird. Das dritte Beispiel ist die Formulierung trp śtt (Caminos 1954, S. 199) trp 'Gänse ${ }^{8}$ rupfen', die in pAnast IV 14, 4 im Zusammenhang mit Aktivitäten rund um die bevorstehende Ankunft des Königs gestanden hat. Das vierte Beispiel ist die Verbindung $r$ 3 hnp $m$ s sttt (Grandet 1994, S. 244, 317) 'gemästete und gerupfte $r$ - Gänse', die aus pHarris ${ }^{9}$ I 16b, 1/pHarris I 65 c, 3 stammt. Das Wort sitt 'rupfen' ist von Piehl (1882, S. 87) für eine Kausativform zu it $\underline{i} i$ gehalten worden, worin ihm Caminos (1954, S. 94) prinzipiell Recht gegeben hat. Im Wörterbuch (WB IV, S. 363, Lemma 1) ist es dagegen als Kausativform (?) wohl zu $\underline{t} t$ 'auflösen' (?) bestimmt worden.

Das zweite hier gemeinte Verb heißt țsí 'binden', das sich in den unterschiedlichsten Kontexten nachweisen lässt. Die verschiedenen Verwendungsmöglichkeiten brauchen hier nicht einzeln durchexerziert werden, da sich der Prozess sonst endlos in die Länge ziehen würde. Um Papier und Tinte zu sparen, reicht es aus zu sagen, dass im hiesigen Fall die Nuance in der Verbindung $\underline{t} s^{\prime} i=f n=k$ śmn (Assmann 2008, S. 476) 'Er (Horus) bindet für dich (Osiris) śmn - Gänse zusammen' von herausragender Bedeutung ist.

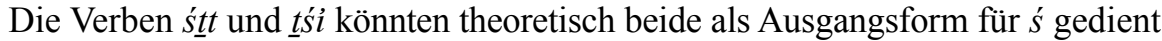
haben, so dass zwischen ihnen nur schwer eine Entscheidung zu treffen ist. Ob die Assimilation von $\underline{t}$ vor oder nach $s$ durchgeführt wird, läuft im Grunde auf das Gleiche hinaus. Wenn śt trupfen' vorzuziehen sein sollte, wäre erst $\underline{t}$ und dann $t$ an $s$ assimiliert und elidiert worden. Die Elision von zwei Konsonanten in der Umgebung von $s$ war auch bei der oben behandelten Schreibung $s$ für $s \underline{d} r$ in Erscheinung getreten. Die Bedeutung $\underline{t} s \dot{i} i$ 'binden' hingegen hätte gegenüber śt t 'rupfen' den kleinen Vorteil, dass sie hier bereits zum zweiten Mal mit der śmn - Gans verbunden wäre. Wenn daher $\underline{t} s \dot{i}$ 'binden' die bessere Alternative ist, wäre nicht nur $\underline{t}$, sondern auch $i$ an $s$ assimiliert

${ }^{5}$ Das auch sonst belegte Wort wi3wi3 hat mehrere Bedeutungen gehabt, die alle negative Züge tragen: zur Wiedergabe mit 'schwach, nachlässig, kraftlos, matt' vgl. Caminos (1963, S. 35); Osing (1998, S. 76h); zur Wiedergabe mit 'ohnmächtig (vornehmlich im Reden)' vgl. Grumach (1972, S. 89); 'ohnmächtig (von Aussprüchen)', vgl. Fischer-Elfert (1986, S. 239). Zur Wiedergabe mit 'unwirksam (vom Bleiglanz)' vgl. Helck (1967, S. 137).

${ }^{6}$ Die gleiche Stelle ist von Foster (1984, S. 93) mit 'You clip the wings (?) of the one who would steal your goods, (Yet) you are ineffectual (sluggish) in my presence' und von Goedicke (1987, S. 65) mit 'You would trust the one who steals your things, (but) you are feeble before me' wiedergegeben worden, die einen etwas anderen Schwerpunkt gesetzt haben.

${ }_{8}^{7}$ pAnast $=$ Papyrus Anastasi.

${ }^{8}$ Zur Ableitung des Gänsenamens von der Wurzel trp 'stumble' und seiner Bestimmung als 'Anser albifrons', vgl. Wolterman (1991-1992, S. 122). Zu diesem Wort vgl. auch Calice (1936, S. 220).

${ }^{9}$ pHarris $=$ Papyrus Harris. 
und elidiert worden. Die zuletzt genannte Assimilation von $i$ an $s$ wäre am einfachsten mit der Ähnlichkeit der beiden Zeichen im Hieratischen zu erklären. Das Wort $s$ 'Gänse drosseln' müsste jedenfalls nach allem, was bisher gesagt worden ist, gestrichen werden.

\section{Bibliographie}

Assmann, J. (2008): Altägyptische Totenliturgien. Band 3: Osirisliturgien in Papyri der Spätzeit. Heidelberg.

Backes, B. (2005): Das altägyptische „Zweiwegebuch“. Studien zu den Sargtext-Sprüchen 10291130. Wiesbaden (Ägyptologische Abhandlungen 69).

Breyer, Fr. (2003): Tanutamani. Die Traumstele und ihr Umfeld. Wiesbaden (Ägypten und Altes Testament 57).

Brunner, H. (1958): Rezension zu: Jaroslav Cerny und Alan H. Gardiner, Hieratic Ostraca I, Oxford, Griffith Institute, 1957, (fol., $\mathrm{x}+35$ pp., 115 plates). Bibliotheca Orientalis Vol. XV, No. 5, pp. 195-197.

Buck, A. de (1957): The Egyptian Coffin Texts III. Texts of Spells 164-267. Chicago, The University of Chicago (Oriental Institute Publications 64).

Calice, Fr. (1936): Grundlagen der ägyptisch-semitischen Wortvergleichung. Eine kritische Diskussion des bisherigen Vergleichsmaterials. Vienna (Beihefte zur Wiener Zeitschrift für die Kunde des Morgenlandes, 1. Heft).

Caminos, R. A. (1954): Late-Egyptian Miscellanies. Bruxelles (Brown Egyptological Studies I).

Caminos, R. A. (1963): Papyrus Berlin 10463, The Journal of Egyptian Archaeology Vol. 49, pp. $29-37$.

Černý, J. (1939): Late Ramesside Letters. Brussels (Bibliotheca Aegyptiaca 9).

Cerny, J. - Gardiner A. H. (1957): Hieratic Ostraca. Vol. 1. Warminster.

El-Kholi, M. S. (2006): Papyri und Ostraka aus der Ramessidenzeit, mit Übersetzung und Kommentar. Siracusa (Monografie del Museo del Papiro 5).

Fecht, G. (1960): Wortakzent und Silbenstruktur. Untersuchungen zur Geschichte der ägyptischen Sprache. Glückstadt-Hamburg-New York (Ägyptologische Forschungen 21).

Fecht, G. (1981): Ptahhotep und die Disputierer (Lehre des Ptahhotep nach Pap. Prisse, Max. 2-4, Dév. 60-83). Mitteilungen des Deutschen Archäologischen Instituts, Abteilung Kairo Vol. 37, pp. 143-150.

Fischer-Elfert, H.-W. (1986): Die satirische Streitschrift des Papyrus Anastasi I., Übersetzung und Kommentar. Wiesbaden (Ägyptologische Abhandlungen 44).

Foster, J. L. (1984): Oriental Institute Ostracon \# 12074. "Menna's Lament" or "Letter to a Wayward Son". The Journal of The Society for the Study of Egyptian Antiquities Vol. 14, pp. $88-89$.

Gardiner, A. H. (1935): Hieratic Papyri in the British Museum. Third Series. Chester Beatty Gift. Volume II, Plates. London.

Gardiner, A. H. (1937): Late-Egyptian Miscellanies. Bruxelles (Bibliotheca Aegyptiaca 7).

Goedicke, H. (1987): Menna's Lament. Revue d'Egyptologie Vol. 38, pp. 63-80.

Grandet, P. (1994): Le Papyrus Harris I (BM 9999). Volume I. Le Caire (Bibliothèque d'Etude $\mathrm{CIX} / \mathrm{I})$.

Grumach, I. (1972): Untersuchungen zur Lebenslehre des Amenope. München-Berlin (Münchner Ägyptologische Studien 23). 
Guglielmi, W. (1983): Eine „Lehre“ für einen reiselustigen Sohn (Ostrakon Oriental Institute 12074). Die Welt des Orients Vol. 14, pp. 147-166.

Helck, W. (1965): Materialien zur Wirtschaftsgeschichte des Neuen Reiches (Teil V), III. Eigentum und Besitz an verschiedenen Dingen des täglichen Lebens, Kapitel AI - AL. Wiesbaden (Akademie der Wissenschaften und der Literatur, Abhandlungen der Geistes- und Sozialwissenschaftlichen Klasse, Jahrgang 1964 - Nr. 4).

Helck, W. (1967): Eine Briefsammlung aus der Verwaltung des Amuntem-pels. Journal of the American Research Center in Egypt Vol. 6, pp. 135-145.

Jansen-Winkeln, K. (2001): Biographische und religiöse Inschriften der Spätzeit aus dem Ägyptischen Museum Kairo. Teil 1: Übersetzungen und Kommentare. Wiesbaden (Ägypten und Altes Testament 45).

Jansen-Winkeln, K. (2007): Inschriften der Spätzeit. Teil II: Die 22.-24. Dynastie. Wiesbaden.

Janssen, J. J. (2008): Daily Dress at Deir el-Medina, Words for Clothing. London (Golden House Publications, Egyptology 8).

Koenig, Y. (1979): Un revenant inconvenant (Papyrus Deir el-Medineh 37). Bulletin de l'Institut Français d'Archéologie Orientale Vol. 79, pp. 103-119.

KRI = Kitchen, Kenneth A. (1983): Ramesside Inscriptions, Historical and Biographical. V. Oxford.

Osing, J. (1998): Hieratische Papyri aus Tebtunis I, Text, The Carlsberg Papyri 2. Copenhagen (CNI Publications 17).

Otto, E. (1960): Das ägyptische Mundöffnungsritual. Teil II: Kommentar. Wiesbaden (Ägyptologische Abhandlungen 3).

Piehl, K. (1882): Dictionnaire du Papyrus Harris $N^{o} 1$ publié par S. Birch: d'après l'original du British Museum. Vienne, Adolf Holzhausen.

Quack, J. Fr. (1999): A New Bilingual Fragment from the British Museum (Papyrus BM EA 69574). The Journal of Egyptian Archaeology Vol. 85, pp. 153-164.

Sethe, K. (1899): Das aegyptische Verbum im Altaegyptischen, Neuaegyptischen und Koptischen. I. Band: Laut- und Stammeslehre. Leipzig.

Sethe, K. (um 1934): Übersetzung und Kommentar zu den altägyptischen Pyramidentexten. III. Band: Spruch 326-435 ( $\$ \$ 534-787)$. Glückstadt-Hamburg-New York.

Sethe, K. (1964): Dramatische Texte zu altaegyptischen Mysterienspielen, II. Der Dramatische Ramesseumpapyrus. Ein Spiel zur Thronbesteigung des Königs. Hildesheim (Untersuchungen zur Geschichte und Altertumskunde Aegyptens 10).

Vernus, P. (1980): Etudes de philologie et le linguistique. Revue d'Egyptologie Vol. 32, pp. 115-134.

WB = Erman, Adolf-Grapow, Hermann (1957): Wörterbuch der aegyptischen Sprache. Vierter Band. 2. Unveränderter Neudruck. Berlin.

Westendorf, W. (1962): Grammatik der medizinischen Texte. Berlin (Grundriss der Medizin der alten Ägypter VIII).

Wolterman, C. (1991-1992): On the Names of Birds and Hieroglyphic Sign - List G 22, G 35 and H 3. Jahrbericht Ex Oriente Lux Vol. 32, pp. 119-130. 